\title{
Response to the letter regarding the original article: the evolution of cardiac point of care ultrasound for the neonatologist
}

\author{
Yogen Singh ${ }^{1,2}(\mathbb{D}$
}

Received: 24 June 2021 / Revised: 24 June 2021 / Accepted: 28 June 2021 / Published online: 7 October 2021

(c) The Author(s), under exclusive licence to Springer-Verlag GmbH Germany, part of Springer Nature 2021

Dear Dr Ibara-Rios et al,

Thank you for your interest in our original article 'The evolution of cardiac point of care ultrasound for the neonatologist' and sharing experience from your institution. I was pleased to hear that you agree with the authors of the original article that the extent of cardiac point of care ultrasound (POCUS) needs to be individualized by the centre needs in close collaboration with Cardiology and Radiology to ensure quality care [1].

Firstly, I must take this opportunity to congratulate your team for establishing hemodynamic consultation program in your clinical practice and adopting this programme in the neonatal fellowship training program. Your experience in performing the Sonographic Algorithm for life threatening Emergencies (SAFE) protocol in the crashing infants demonstrates why such protocols need adopting in the neonatal clinical practice. You might be interested in our recently published experience from adopting SAFE-R (Sonographic Assessment of liFe-threatening Emergencies-Revised) protocol, which is based upon integration of our current knowledge on the use of POCUS for diagnosis of the most critical neonatal complications. The main features of SAFE-R are the use of standardized ultrasound points, and a simple one-probe rule-in/rule-out approach [2].

Secondly, as we discussed in our original paper, cardiac POCUS is different from a protocol-based neonatologist performed comprehensive echocardiography. Cardiac POCUS, neonatologist performed echocardiography, and a formal echocardiography performed by a pediatric cardiologist or trained

Communicated by Daniele De Luca

This reply refers to the comment available online at https://doi.org/ 10.1007/s00431-021-04183-z.

Yogen Singh

Yogen.Singh@nhs.net

1 Department of Pediatrics - Neonatology and Pediatric Cardiology, Cambridge University Hospitals, NHS

Foundation Trust, Cambridge, UK

2 Loma Linda University School of Medicine, Loma Linda, CA, USA sonographer involve different levels of cardiac imaging assessment. The three modalities are the continuum of cardiac imaging and require different training to gain competencies in performing these assessments. Clinician performing ultrasonography needs to understand their indications and limitations $[1,3]$.

Lastly, international evidence-based guidelines on POCUS for critically ill neonates and children issued by the POCUS Working Group of the European Society of Paediatric and Neonatal Intensive Care (ESPNIC), your experience and SAFE-R protocol show that use of POCUS is not limited to cardiac assessment in neonates and its role is fast evolving in neonatal clinical practice, especially in the sick infants to answer specific question and help the clinicians in making a timely and accurate clinical decision on the bedside. However, now there is an urgent need of developing a POCUS training curriculum and certification process so that its use in clinical practice can be standardized [3].

\section{Declarations}

Conflict of interest The author declares no competing interests.

\section{References}

1. Singh Y, Bhombal S, Katheria A, et al. The evolution of cardiac point of care ultrasound for the neonatologist. Eur J Pediatr (2021 - published online ahead of publication). https://doi.org/10.1007/ s00431-021-04153-5

2. Yousef N, Singh Y, De Luca D. "Playing it SAFE in the NICU" SAFE-R: a targeted diagnostic ultrasound protocol for the suddenly decompensating infant in the NICU. Eur J Pediatr 2021 (accepted for publication).

3. Singh Y, Tissot C, Fraga MV, et al. International evidence-based guidelines on Point of Care Ultrasound (POCUS) for critically ill neonates and children issued by the POCUS Working Group of the European Society of Paediatric and Neonatal Intensive Care (ESPNIC). Crit Care 2020; 24: 24(1):65. https://doi.org/10.1186/ s13054-020-2787-9.

Publisher's Note Springer Nature remains neutral with regard to jurisdictional claims in published maps and institutional affiliations. 